\title{
44528 - DOES THE APOE-E4 GENOTYPE PREDICT DELIRIUM OR POCD AFTER AORTIC REPAIR?
}

\author{
Gregory Lyle Bryson, The Ottawa Hospital - Civic Campus, Ottawa, ON, Canada; \\ Anna Wyand, The Ottawa Hospital - Civic Campus; \\ Wozny Denise, The University of Ottawa Heart Institute; \\ LM Rees, The Ottawa Hospital Rehabilitation Centre; \\ L Vasudev, Élisabeth Bruyère Health Centre; \\ HJ Nathan, The University of Ottawa Heart Institute;
}

INTRODUCTION: Failure of cholinergic neurotransmission is felt to be central to the mechanism of delirium(1) and progressive dementias such as Alzheimer's disease and vascular dementia(2). It has been suggested that low concentrations of apolipoprotein $\mathrm{E}$ (APOE) in the brains of individuals with the APOE-E4 genotype compromise phospholipid metabolism resulting in selective impairment of cholinergic neurotransmission(3). The purpose of this study was to determine if patients expressing the APOE-E4 genotype were at increased risk of either delirium or postoperative cognitive dysfunction (POCD) following aortic aneurysm repair.

METHODS: Following research ethics board approval and written informed consent a cohort of patients 60 years of age and older undergoing open repair of the abdominal aorta was recruited. APOE genotyping and a battery of nine neuropsychometric (NP) tests were performed prior to surgery. Drugs with significant psychoactive properties and patients with pre-existing dementia were excluded. Delirium was assessed on postoperative days (POD) 2, 4 and 7 using the Confusion Assessment Method (CAM)(4). NP testing was repeated on POD7 and again 3 months following surgery. NP testing results from a group of non-surgical patients was used to adjust NP scores using reliable change index methodology(5). POCD was diagnosed if a 2 standard deviation decrease in NP testing was detected.

RESULTS: Fifty-nine patients have thus far undergone preoperative NP testing and surgery. Seven patients (4 deaths, 3 withdrawals) precluded complete in-hospital testing for all patients. Patient characteristics and cognitive outcomes are highlighted in Table 1. Fourteen (74\%) patients experiencing delirium were noted to have POCD at POD7 $(\mathrm{p}=0.041)$. APOE genotyping was available for 44 patients. Delirium developed in 4 of 9 patients $(44 \%)$ with the APOE-E4 genotype $(\mathrm{p}=0.625)$ while POCD at POD7 was noted in 8 of $9(89 \%)$ of those same patients ( $\mathrm{p}=0.107)$. To date, 2 of 38 patients $(5.3 \%)$ assessed at 3-months had persistent POCD, one of whom expressed the APOE-E4 genotype. 


\section{Table 1. Patient Characteristics and Outcomes}

\begin{tabular}{|l|c|}
\hline \multicolumn{2}{|c|}{ Characteristic } \\
\hline Age & $71(6)$ \\
Gender (M/F) & $44 / 15$ \\
MMSE at baseline & $28.6 \pm 1.2$ \\
\hline APOE Genotype & $1(2)$ \\
\hline \multicolumn{1}{|l|}{ E4 - E4 } & $9(20)$ \\
E4 - Other & $34(78)$ \\
Other - Other & $19(36)$ \\
Delirium & $33(64)$ \\
POCD & $4(7)$ \\
Death & $12.5 \pm 12.2$ \\
\hline Length of stay (days)
\end{tabular}

Discussion: Delirium and POCD were common following open aortic repair.

Perioperative delirium appears to predict POCD at POD7. The APOE-E4 genotype was not associated with delirium but was more frequent in those developing POCD. The association between APOE-E4 and POCD did not reach statistical significance. POCD persisting 3 months following surgery was infrequent.

References: Semin Clin Neuropsychiatry 2000; 5(2):132-148. 2. J Neurol Sci 2002; 203204:131-136. 3.Trends Neurosci 1994; 17(12):525-530. 4. Ann Intern Med 1990; 113(12):941-948. 5. J Consult Clin Psychol 1991;59:12-9 